University of Nebraska - Lincoln

DigitalCommons@University of Nebraska - Lincoln

$4-2009$

\title{
Prey capture by the whip spider Phrynus marginemaculatus C.L. Koch
}

Roger D. Santer

University of Nebraska - Lincoln, roger.santer@ul.ie

Eileen Hebets

University of Nebraska - Lincoln, ehebets2@unl.edu

Follow this and additional works at: https://digitalcommons.unl.edu/bioscihebets

Part of the Behavior and Ethology Commons

Santer, Roger D. and Hebets, Eileen, "Prey capture by the whip spider Phrynus marginemaculatus C.L. Koch" (2009). Eileen Hebets Publications. 33.

https://digitalcommons.unl.edu/bioscihebets/33

This Article is brought to you for free and open access by the Papers in the Biological Sciences at DigitalCommons@University of Nebraska - Lincoln. It has been accepted for inclusion in Eileen Hebets Publications by an authorized administrator of DigitalCommons@University of Nebraska - Lincoln. 


\title{
SHORT COMMUNICATION
}

\section{Prey capture by the whip spider Phrynus marginemaculatus C.L. Koch}

\author{
Roger D. Santer ${ }^{1}$ and Eileen A. Hebets: School of Biological Sciences, University of Nebraska-Lincoln, Lincoln, \\ Nebraska 68588, USA. E-mail: roger.santer@ul.ie
}

\begin{abstract}
Whip spiders (Arachnida, Amblypygi) are little-studied arachnids with enlarged spiny pedipalps and elongated "antenniform" forelegs. These antenniform legs contain at least seven giant sensory neurons with no known behavioral function. Here we use high-speed cinematography to describe the prey capture behavior of the whip spider Phrynus marginemaculatus C.L. Koch 1840, in order to examine how these giant neurons might be involved. When presented with a prey item (a cricket), a whip spider first accurately aims one of its antenniform legs in the prey's direction. Next, the whip spider orients its body to the prey item and approaches, placing one antenniform leg tip on either side of the prey. The whip spider may remain relatively still in this position for some time, before opening its pedipalps in preparation for a strike and then rapidly swinging its antenniform legs away from the prey item and striking at it with its pedipalps. In common with previous studies, our results show that giant neuron activity is not necessary to trigger any of the stages of normal prey capture behavior, but they also suggest that these neurons could still provide information important in this context.
\end{abstract}

Keywords: High-speed film, predator, attack

Whip spiders (Arachnida, Amblypygi) possess a number of morphological specializations including enlarged spiny pedipalps and elongated antenniform forelegs which they use as feelers. These antenniform legs are equipped with a variety of sensory organs (Igelmund 1987; Weygoldt 2000), and mechanosensory information from some of these organs is rapidly transmitted to the central nervous system by at least seven identified giant neurons (GNs) (Igelmund \& Wendler 1991a). The response properties of four of these neurons are now known: interneurons GN1 and 2 are mechanosensory and respond to mechanical contacts with the bristle hairs on the antenniform leg tarsus; sensory neurons GN6 and 7 are proprioceptors that detect bending of the tarsus around a particular joint (Igelmund \& Wendler 1991a, b). The behavioral function of these giant neurons is unknown, but their presence in whip spiders from very different habitats indicates that they may play a role in fundamental behavior (Spence \& Hebets 2007); one suggestion has been that they function in prey capture (see Weygoldt 2000).

The antenniform legs, and their GNs, are not necessary for successful prey capture since a whip spider that has autotomized both of these limbs can still capture prey (Beck \& Görke 1974; Weygoldt 1995, 2000). In contrast, removal of the trichobothria - air movement-sensitive hairs predominantly located on the walking legs - leaves a whip spider unable to orient towards or capture moving prey (Beck \& Görke 1974; Weygoldt 1995, 2000), demonstrating that these hairs are necessary and sufficient for successful prey capture. Nevertheless, this evidence does not preclude a secondary role for the GNs in prey capture when they are intact, and field observations suggest that they might play a role in the capture of aquatic prey during the fishing behavior of the whip spider Heterophrynus cheiracanthus Gervais 1842 (Ladle \& Velander 2003). In this note we use high-speed cinematography to examine the possible role of the GNs in the capture of terrestrial prey and to provide a detailed kinematic description of this behavior.

We collected Phrynus marginemaculatus C.L. Koch 1840 from the Pine Rock hammock on Big Pine Key, FL, USA $\left(24^{\circ} 42^{\prime} 33.49^{\prime \prime} \mathrm{N}\right.$, $81^{\circ} 22^{\prime} 56.73^{\prime \prime} \mathrm{W}$ ), and housed them in our laboratory under a $12: 12 \mathrm{~h}$ light-dark cycle. Voucher specimens have been deposited in the collection of the University of Nebraska State Museum (accession

${ }^{1}$ Current address: Department of Life Sciences, University of Limerick, Limerick, Ireland. number: 272; specimen numbers: 3257774, 3257775). We performed experiments on six adult female whip spiders since we used the males we collected in an unrelated study (Santer \& Hebets 2008). However, informal observations of the feeding behavior of these males revealed no differences from that of females. In order to allow prey capture behavior to be filmed, we permanently housed whip spiders in cages $(10 \mathrm{~cm} \times 10 \mathrm{~cm} \times 11 \mathrm{~cm})$ custom built from sheets of clear acetate. Aluminum screening was placed on the rear wall of all cages, providing a surface upon which the animals could climb. Whip spiders would remain on the screening, allowing a prey item to be introduced and prey capture behavior to be filmed, without transferring the whip spider to an experimental arena. We introduced a prey item once every two weeks during the light phase of the light cycle and filmed the resulting predator-prey interaction at 60 or 250 fps from two angles (cage front and side) using a Photron Fastcam 1024 PCI camera and mirror.

When collecting $P$. marginemaculatus we commonly found them alongside numerous cockroaches, small scorpions, and centipedes. We therefore believe that ground dwelling invertebrates form the bulk of $P$. marginemaculatus' diet. In the laboratory, two-week old crickets were readily attacked and eaten and so we use them as a typical prey item in this study. Little data are available on the natural diet of whip spiders, but their main food is thought to consist principally of arthropods, particularly insects (Weygoldt 2000). Crickets and cockroaches are a known part of the diet of a related whip spider species, Phrynus pseudoparvulus Armas \& Víquez 2001 (previously thought to be Phrynus parvulus, Armas \& Víquez 2001), for which feeding data are available (Hebets 2002). Phrynus pseudoparvulus has also been observed feeding on moths captured in flight (Hebets 2002), but when we presented P. marginemaculatus with moths collected locally in Lincoln, NE, USA, we found that moths were rarely attacked and that the whip spiders were often startled by a moth's flapping movements. This may indicate that moths are not a typical part of $P$. marginemaculatus' diet or that the species presented were inappropriate.

In total, we successfully filmed 27 high-speed video sequences in which a whip spider attacked a cricket by striking at it with its pedipalps (minimum of one prey capture per whip spider). Across these sequences, prey capture behaviors were remarkably similar and preparatory behaviors comprised a sequence of three distinct behavioral actions: 

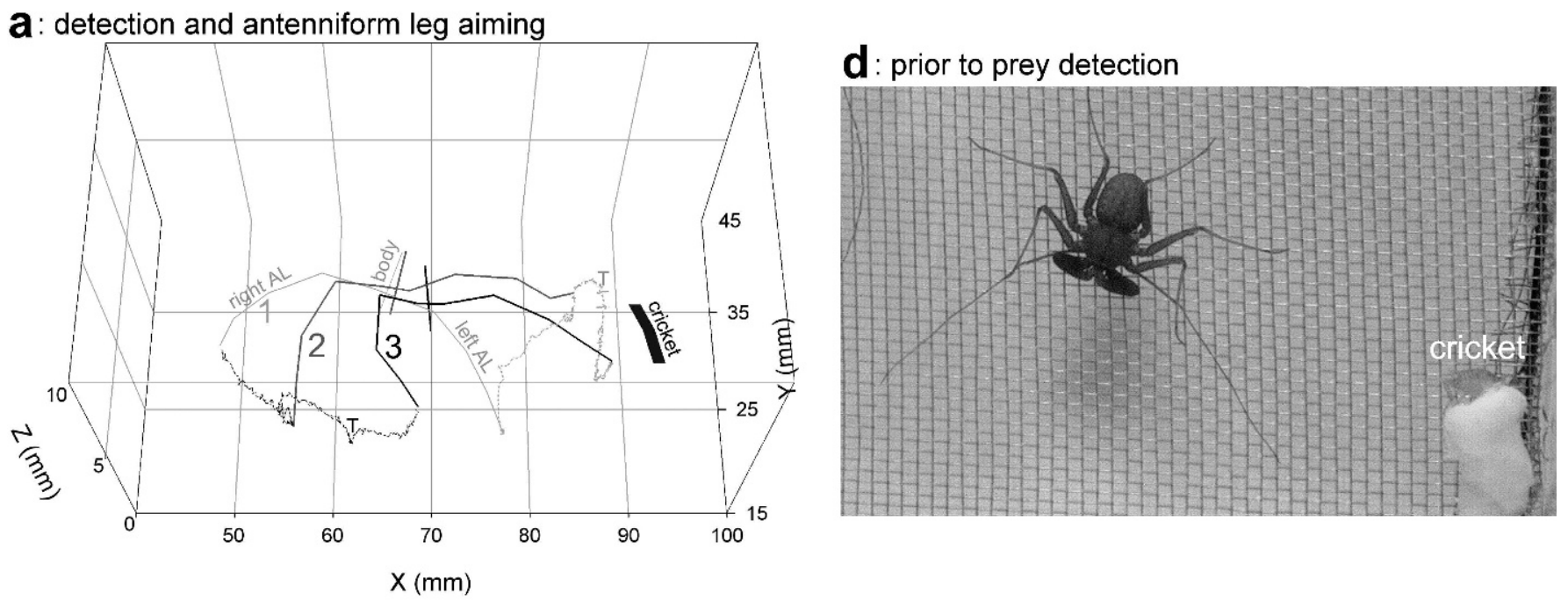

b: body orientation and approach

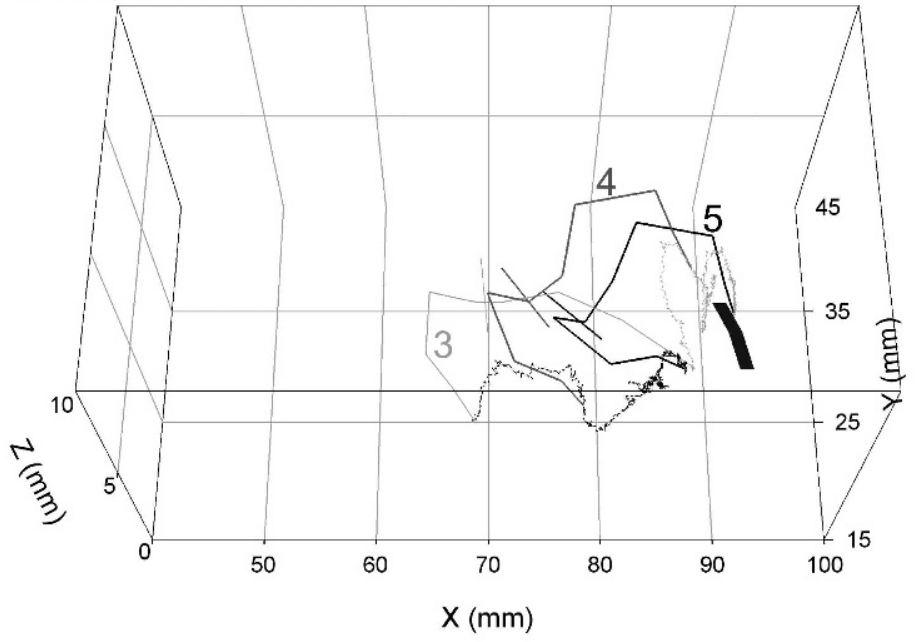

\section{e: antenniform leg aiming}

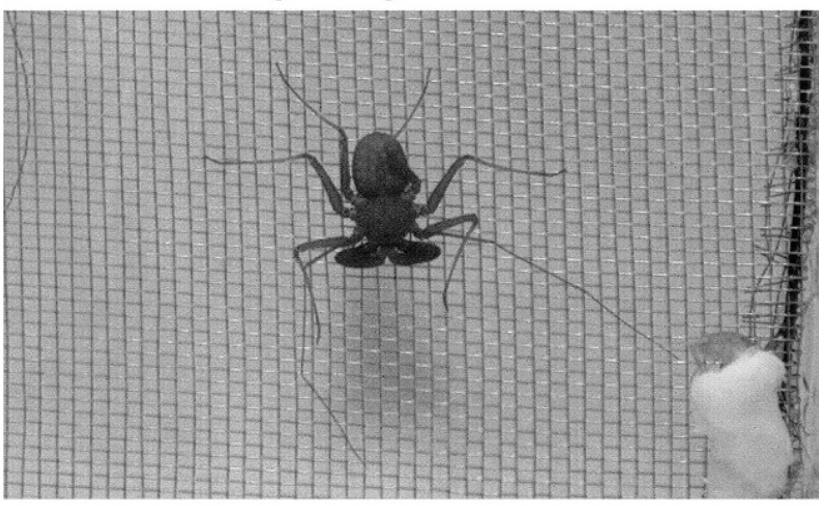

\section{C : pre-strike examination}

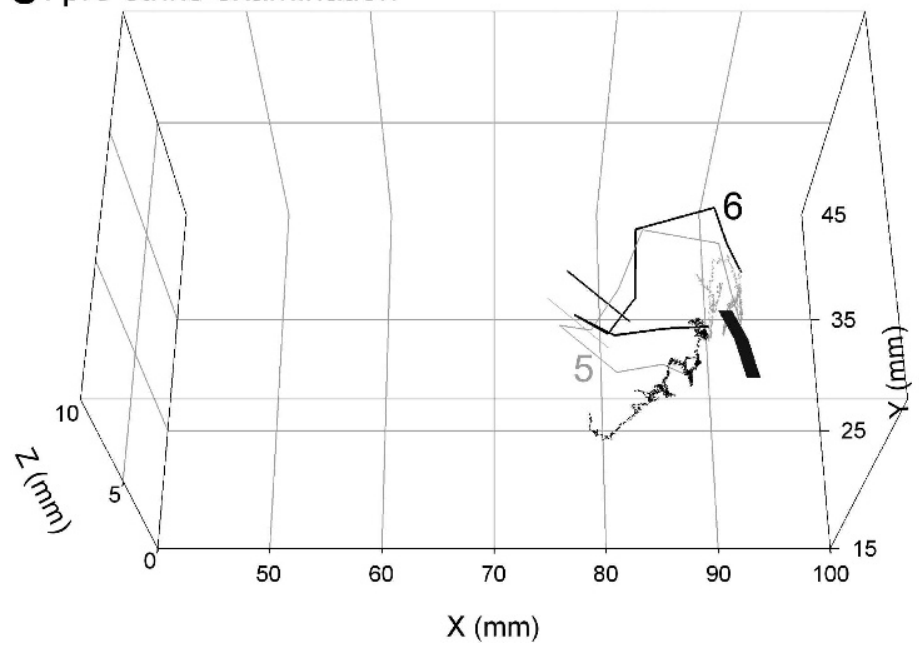

f: pre-strike examination

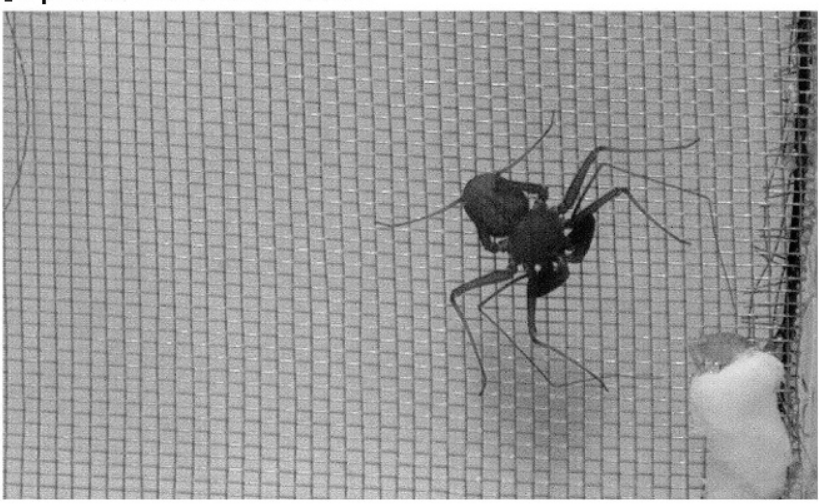

Figure 1.-The typical sequence of preparatory behaviors preceding a prey capture strike in Phrynus marginemaculatus. Panels a-c describe the three principal behavioral phases transcribed from each frame of a typical prey capture sequence recorded at $60 \mathrm{fps}$. In each panel, the whip spider's body and antenniform leg positions are plotted at intervals as solid lines (labeled "left AL," "body," "right AL" in panel a). The grayscale of these lines and the associated number indicates their position in the sequence $(1=0 \mathrm{~ms}, 2=2000 \mathrm{~ms}, 3=5500 \mathrm{~ms}, 4=7167 \mathrm{~ms}, 5$ $=15500 \mathrm{~ms}, 6=23333 \mathrm{~ms}$ ). In addition, left and right antenniform leg tip positions are plotted at each frame (light gray and black jagged traces 
(1) Prey detection and antenniform leg aiming.--After a cricket was released into its cage, the first notable action by the whip spider was to aim one or other of its antenniform legs in the direction of the prey item without re-orientation of its body (Figs. 1a, e). This action occurred in $88.9 \%$ of filmed feeding events (the remainder resulting from the cricket actually walking into the whip spider apparently before detection). Since the walking leg trichobothria are necessary and sufficient for successful prey capture (Beck \& Görke 1974; Weygoldt 1995), it seems likely that they could also be responsible for initial prey detection. The trichobothria could provide the necessary directional information for antenniform leg aiming (e.g., Friedel \& Barth 1997). Antenniform leg aiming could last indefinitely in trials where the cricket was not ultimately attacked, or until the next phase of the prey capture sequence occurred when it was.

(2) Body orientation and approach.-Following a period of antenniform leg aiming, whip spiders re-oriented their bodies towards the cricket or slowly approached it until it was within the tips of the antenniform legs and normally until the long axis of their body was in line with the cricket (Fig. 1b). During this phase, the antenniform leg tips sometimes (in $55.6 \%$ of trials) made repeated gentle contacts with the cricket that may have been attempts at chemical examination; these contacts did not startle the cricket. In cases where the cricket walked into the antenniform leg, the antenniform leg was withdrawn rapidly (see also Foelix \& Troyer 1980). Body orientation and approach occurred in $85.2 \%$ of the filmed feeding trials.

(3) Pre-strike prey examination.-The final preparatory action before a strike was for the whip spider to place its left and right antenniform legs on either side of the prey item, usually without making contact with it (Figs. 1c, f). This behavior may be an attempt to examine the odor of the prey item (Hebets \& Chapman 2000). Such behavior occurred in $77.8 \%$ of trials, although in the remainder of trials examination by one antenniform leg only often occurred. During prey examination, the left antenniform leg tip was placed 2.97 $\pm 0.59 \mathrm{~mm}$ from the nearest part of the cricket's body or appendage (range: $0.00-9.00 \mathrm{~mm}$ ), and the right antenniform leg $3.13 \pm 0.65 \mathrm{~mm}$ from it (range: $0.00-12.87 \mathrm{~mm})(n=27$ in both cases; measurements made immediately prior to a prey capture strike; here and throughout means \pm SEM). From the 27 filmed prey capture sequences, eight could have included fleeting contact between the whip spider's antenniform leg and the limbs, antennae, or cerci of the cricket during pre-strike examination. Whether contact occurred in these trials could not be firmly established from the recorded videos. In the 21 trials where examination of the prey item using both antenniform legs occurred, it had a mean duration of $3.74 \pm 1.15 \mathrm{~s}$ (range: 0.04 $21.33 \mathrm{~s})(n=21)$.

Following these preparatory behaviors, a whip spider struck at its prey using its pedipalps. The prey capture strike was remarkably stereotyped between trials. Following pre-strike prey examination, the pedipalps were slowly opened, the chelicerae extended, and the whip spider rapidly rocked forwards on its six walking legs. Normally these legs maintained contact with the screened cage wall (where the whip spider usually stood), but sometimes it "jumped." During this strike, the antenniform legs were rapidly swung outwards and rearwards from their pre-strike examination positions, presumably to ensure that they were not damaged by the strike. Strikes were initiated with the prey item at a mean distance of $14.03 \pm 0.70 \mathrm{~mm}$ from the chelicerae (range: $6.77-20.21 \mathrm{~mm})(n=27)$. Strikes covered this distance with a mean speed of $0.17 \pm 0.02 \mathrm{~ms}^{-1}$ (range: 0.008 $0.326 \mathrm{~ms}^{-1}$ ). However, often the strike itself would consist of two phases: an initially slow approach lunge phase followed by an extremely fast one. Mean maximum acceleration (measured frame by frame from each recording) was $18.74 \pm 3.12 \mathrm{~ms}^{-2}$ (range: $3.09-$ $59.37 \mathrm{~ms}^{-2}$ ).

Our high-speed video sequences revealed no reliable associations between mechanical contacts that might excite the GNs and the typical stages of prey capture behavior. For example, the antenniform leg tips are held very close to a potential prey item during pre-strike examination but because physical contact is unusual, the mechanosensory neurons GN1, 2, 6 and 7 cannot be necessary to trigger a prey capture strike (see also Foelix \& Hebets 2001). Thus GN activity cannot be responsible for triggering the typical sequence of prey capture behaviors, as indicated by previous studies (Beck \& Görke 1974; Weygoldt 1995, 2000). Nevertheless, our results do suggest that the GNs have an important role to play in supplying sensory information for prey capture behavior.

Firstly, the antenniform legs sometimes repeatedly contacted the cricket during body orientation and approach, but did not startle it. A fast conducting mechanoreceptive neuron like GN1 would be needed to ensure that these contacts were sufficiently gentle.

Secondly, since the antenniform leg tips are placed on either side of the prey item during pre-strike examination, any movement of the prey item would contact the antenniform leg and excite GN1 or 2. Although this activity is not necessary for prey capture, we did see instances where it could have alerted the whip spider to a sudden and unpredicted prey movement and where it was immediately followed by a strike.

Finally, in one video sequence we noted that the whip spider lost the position of its prey item during antenniform leg aiming. The cricket then approached the whip spider apparently undetected from behind and made contact with one of its antenniform legs. This triggered a rapid re-orientation by the whip spider followed by a sequence of pre-strike examination. The re-orientation began less than $16.6 \mathrm{~ms}$ (one frame) after contact by the cricket and contact occurred with the area of the antenniform leg tarsus from which GN2 receives excitation. From here, impulses have approximately $28 \mathrm{~mm}$ to travel to the central nervous system and, using a conduction velocity of $2.6 \mathrm{~ms}^{-1}$ for GN2 (Spence \& Hebets 2007), they could cover this distance in $10.8 \mathrm{~ms}$. Thus this re-orientation is likely to have been GN2-mediated since neurons of smaller diameter could not convey impulses sufficiently rapidly. On several occasions the antenniform leg was rapidly withdrawn if the cricket contacted it, and these movements may also have been GN-mediated (see also Foelix \& Troyer 1980). If the function of the GNs were highly context dependent, this could explain why motor responses were not previously found to be reliably associated with GN activity (Igelmund \& Wendler 1991b).

In this note we have described the prey capture behavior of the whip spider P. marginemaculatus. We confirmed that the GNs were

$\leftarrow$

labeled " $T$ " in panel a). In this sequence the whip spider is preparing to attack a cricket. The cricket's position is plotted as a thick black line (marked "cricket" in panel a). Panels $\mathbf{d}-\mathbf{f}$ are example frames from the high-speed video sequence from which panels a-c were transcribed. In each frame the cricket sits on a moistened cotton wick in the bottom right corner of the frame ("cricket" in panel d). Typically, when a prey item is detected, a whip spider aims its antenniform leg at it without reorienting its body $(0-5500 \mathrm{~ms}$; a). The whip spider's antenniform leg positions before and during antenniform leg aiming are illustrated in frames $\mathbf{d}$ and e. Following antenniform leg aiming, the whip spider re-orients and approaches the prey item, placing one antenniform leg tip on either side of it (5500-15500 ms; b). The whip spider remains relatively still with its antenniform leg tips either side of the prey item, but not usually contacting it, during a phase of pre-strike examination (15500-23333 ms; c). Typical antenniform leg positions at the onset of pre-strike examination are illustrated in frame f. Following pre-strike examination, a prey capture strike is initiated (see text). 
not necessary for triggering any of the typical stages of prey capture, but our data did indicate several supporting roles that the GNs might play in this context. Future study will be necessary to understand these roles and why GNs have evolved in whip spiders.

\section{ACKNOWLEDGMENTS}

We thank the U.S. Fish and Wildlife Service and National Key Deer Refuge for permitting whip spider collection; and R.H. Willemart for comments and discussion. Funding was from a Searle Foundation Scholar grant to EAH.

\section{LITERATURE CITED}

Armas, L.F. de. \& C. Víquez. 2001. Nueva especie de Phrynus (Amblypygi: Phrynidae) de Costa Rica. Revista Ibérica de Aracnología 4:11-15.

Beck, L. \& K. Görke. 1974. Tagesperiodik, Revierverhalten und Beutefang der Geisselspinne Admetus pumilio C.L. Koch im Freiland. Zeitschrift für Tierpsychologie 35:173-186.

Foelix, R.F. \& E.A. Hebets. 2001. Sensory biology of whip spiders (Arachnida, Amblypygi). Andrias 15:129-140.

Foelix, R.F. \& D. Troyer. 1980. Giant neurons and associated synapses in the peripheral nervous system of whip spiders. Journal of Neurocytology 9:517-535.

Friedel, T. \& F.G. Barth. 1997. Wind-sensitive interneurones in the spider CNS (Cupiennius salei): directional information processing of sensory inputs from trichobothria on the walking legs. Journal of Comparative Physiology A 180:223-233.

Hebets, E.A. 2002. Relating the unique sensory system of amblypygids to the ecology and behavior of Phrynus parvulus from Costa Rica (Arachnida, Amblypygi). Canadian Journal of Zoology 80:286-295.
Hebets, E.A. \& R.F. Chapman. 2000. Electrophysiological studies of olfaction in the whip spider Phrynus parvulus (Arachnida, Amblypygi). Journal of Insect Physiology 46:1441-1448.

Igelmund, P. 1987. Morphology, sense organs and regeneration of the forelegs (whips) of the whip spider Heterophrynus elaphus (Arachnida, Amblypygi). Journal of Morphology 193:75-89.

Igelmund, P. \& G. Wendler. 1991a. The giant fiber system in the forelegs (whips) of the whip spider Heterophrynus elaphus Pocock (Arachnida: Amblypygi). Journal of Comparative Physiology A 168:63-73.

Igelmund, P. \& G. Wendler. 1991b. Morphology and physiology of peripheral giant interneurons in the forelegs (whips) of the whip spider Heterophrynus elaphus Pocock (Arachnida: Amblygi). Journal of Comparative Physiology A 168:75-83.

Ladle, R.J. \& K. Velander. 2003. Fishing behavior in a giant whip spider. Journal of Arachnology 31:154-156.

Santer, R.D. \& E.A. Hebets. 2008. Agonistic signals received by an arthropod filiform hair allude to the prevalence of near-field sound communication. Proceedings of the Royal Society B Biological Sciences 275:363-368.

Spence, A.J. \& E.A. Hebets. 2007. Anatomy and physiology of giant neurons in the antenniform leg of the amblypygid Phrynus marginemaculatus. Journal of Arachnology 34:566-577.

Weygoldt, P. 1995. A whip spider that ate rolled oats, with observations on prey-capture behaviour in whip spiders. Newsletter of the British Arachnological Society 74:6-8.

Weygoldt, P. 2000. Whip Spiders (Chelicerata: Amblypygi). Their Biology, Morphology and Systematics. Apollo Books, Stenstrup, Denmark. 168 pp.

Manuscript received 18 December 2007, revised 15 August 2008. 